Original Research Paper

\title{
Overdominance Effects between Malaria and Visceral Leishmaniasis in the 5 q31 Region
}

\author{
${ }^{1}$ Mutaz Amin, ${ }^{1}$ Abdelbadea Elhassan, ${ }^{2}$ Kirk Rocket and ${ }^{1}$ Muntasir Ibrahim \\ ${ }^{I}$ Department of Molecular Biology, Institute of Endemic Diseases, University of Khartoum, Khartoum, Sudan \\ ${ }^{2}$ Wellcome Trust Centre for Human Genetics, University of Oxford, Roosevelt Drive, Oxford, UK
}

Article history

Received: 27-02-2015

Revised: 08-07-2015

Accepted: 03-08-2015

Corresponding Author: Muntasir Ibrahim

Department of Molecular

Biology, Institute of Endemic

Diseases, University of

Khartoum, Khartoum, Sudan

Email: mibrahim@iend.org

\begin{abstract}
A group of SNPs in 5q31, genotyped from Hausa tribe of Koka village in eastern Sudan; an area endemic with malaria and visceral leishmaniasis, were analyzed by The Leishmaniasis research group and found to have significant excess of heterozygosis level and departure from HWE and assumed to be due to natural selection resulting from notorious deadly outbreaks since various ethnic groups from western Sudan settled the area although sequence information in the $5 \mathrm{q} 31$ region did not detect functional SNPs that could be associated with such drastic phenotypes. Malaria was thought to inflict inferior selective pressure due to the mild clinical phenotype observed. Taking advantage of follow up phenotype data sets available from the Malaria Gen study we re-analyzed these genotypes using different bioinformatics software to determine their effect on the regulation, function and expression of interleukins, miRNA binding and splicing mechanism. The SNPs were found to potentially affect the binding of many transcription factors that regulate the expression of IL-4 and IL-13 and stability of IL-5 mRNA. Association of haplotypes susceptibility revealed that the haplotype of low cytokine $\mathrm{TH} 2$ profile is associated with higher risk of malaria infection (P-value $=0.02$ ). Through modulating TH2 cytokine response; the excess heterozygosity in $5 \mathrm{q} 31$ is explained with the phenomenon of overdominance between malaria and visceral leishmaniasis, acted by natural selection and driving the locus towards optimum response.
\end{abstract}

Keywords: Heterozygosity, Natural Selection, Single Nucleotide Polymorphisms, Overdominance

\section{Introduction}

The $5 \mathrm{q} 31$ in chromosome 5 is of much interest because it encompasses genes of type 2 cytokine responses known to associate with a number of infectious and noninfectious diseases and the fact that a quantitative trait locus associated with inflammatory diseases has been mapped in this region. The region contains several genes that modulate atopic responses, including IL-3, IL-4, IL-13, IL-5, CD14 and granulocyte macrophage-colony-stimulating factor, which are involved in the control of immunity to $P$. falciparum blood stages (Troye-Blomberg et al., 1990) and susceptibility to visceral leishmaniasis (Mohamed at al., 2003). Elhassan et al. (2013) suggested that the region is associated with signals of natural selection in areas endemic with malaria and visceral leishmaniasis. They selected two sets of Single Nucleotide Polymorphisms (SNPS) within the genes of IL-4, IL-5 IL-9 and IL-13, in
57 unrelated individuals. The first subset (4 SNPs) showed significant increase in heterozygosis level and departure from HWE, the second subset (14 SNPs) used to generate haplotype blocks (Elhassan et al., 2013).

The studied areas are Koka and Um-Salala villages located in the eastern bank of Rahad River in eastern Sudan; inhabited by Hausa and Massalit tribes respectively. The outbreaks of VL in the eighties and nineties in southern Sudan resulted in probably some of the most severe mortality indexes in modern history with hundreds of thousands reported to have succumbed to the disease (Elhassan et al., 2013).

Elhassan et al. (2013) suggested that the $5 \mathrm{q} 31$ area to be under intense selective pressure as indicated by marked heterozygosity independent of Linkage Disequilibrium (LD); difference in heterozygosity, allele and haplotype frequencies between generations and departure from Hardy-Weinberg Expectations (HWE) (Elhassan et al., 2013). 
The objective of this study is to try to find out the underlying mechanism of this observed heterozygosity by analyzing the effect of these SNPs on the structure, function, stability and regulation of interleukins using software bioinformatics techniques. We will measure the susceptibility of different genotypes and haplotypes to malaria focusing on Hausa tribe of Koka village because they have clearer genotypes data. Understanding such phenomena will be important for learning the complex interaction between infectious diseases and immunity and the factors that drive human evolution.

\section{Materials and Methods}

A data set comprising information on age, gender; genotypes of SNPs in IL-4, IL-5, IL-9 and IL-13, of 57 unrelated subjects from Hausa tribe published previously (Elhassan et al., (2013) were reanalyzed with updated information regarding malaria affection status (positive or negative by ICT) and serum IgE level. The SNPs studied, heterozygosity level and HWE is shown in Table 1. The SNP rs1799962 in IL-9 is excluded from subsequent analysis because its heterozygosity level was found statistically non-significant (Elhassan et al., 2013). The list of bioinformatics tools used in subsequent analyses is shown in Table 2.

\section{Results}

Malaria infection was more common in children $(\leq 15$ years) than adults ( $>15$ years), but the result was statistically non-significant $(\mathrm{p}$-value $=0.09$ ). Association between malaria/gender and malaria/serum Ig-E level was nonsignificant either ( $\mathrm{p}$-value $=0.24$ and 0.07 respectively).

The SNP rs1800474 in IL-5 (heterozygotes) was significantly protective against malaria ( $\mathrm{p}$-value = 0.008), compared to IL-4 (rs734244) and IL-13 (rs1881457), $\mathrm{p}$-value $=0.11$ and 0.24 respectively, Fig. $1 \mathrm{a}, 1 \mathrm{~b}$ and $1 \mathrm{c}$. The results' significance persisted after permutation. The haplotype GCG of IL-4, IL-13 and IL5 respectively was associated with significantly lower risk of malaria infection, Table 3.

The SNPs rs734244, rs 1881457 and rs2070874 (the latter is found to be in perfect LD to rs734244 in YRI population) were found to affect many transcription factor binding sites, the transcription factors and binding scores varied between different software tools, Table 4 . The effect of SNPs on transcription factor binding was verified using Alibaba 2.1 and TFSEARCH tools.rs 1800474 was found to affect the thermodynamic stability of IL-5 mRNA by changing the free energy of the molecule from- $189.3 \mathrm{kcal} / \mathrm{mol}$ to- $191.9 \mathrm{kcal} / \mathrm{mol}$.

Table 1. Shows the studied SNPs, located genes, position and variation, observed and expected heterozygosity and p-value for DHWE

\begin{tabular}{lllllll}
\hline SNP & Gene & Position & Var & Obs Hz & Exp Hz & $p$-value \\
\hline rs734244 & Il-4 & Intronic & A/G & 0.85 & 0.49 & $2.8 \mathrm{E}-9$ \\
rs1800474 & Il-5 & Exonic & A/G & 0.71 & 0.45 & $4.9 \mathrm{E}-5$ \\
rs1799962 & Il-9 & Intronic & A/G & 0.48 & 0.38 & 0.09 \\
rs1881457 & IL-13 & Promoter & A/C & 0.56 & 0.40 & 0.014 \\
\hline
\end{tabular}

Table 2. Shows the list of bioinformatics tools used in this article, their functions and websites

\begin{tabular}{lll}
\hline Software & Function & website \\
\hline SNP function prediction & Transcription factor binding & $\mathrm{http}: / /$ genome.ucsc.edu/). \\
Is-rSNP & Transcription factor binding & $\mathrm{http} / / /$ bioinformatics.research.nicta.com.au/software/is-rsnp/ \\
RegulomeDB & Transcription factor binding & $\mathrm{http} / / /$ regulomedb.org/ \\
r-SNPBase & Transcription factor binding & $\mathrm{http}: / /$ rsnp.psych.ac.cn/ \\
AliBaba2.1 & Transcription factor binding & $\mathrm{http}: / /$ labmom.com/link/alibaba_2_1_tf_binding_prediction \\
TFSEARCH & Transcription factor binding & $\mathrm{http}: / /$ diyhpl.us/ bryan/irc/protocol-online/protocol-cache/TFSEARCH.html \\
mirSNP & mi-RNA binding prediction & $\mathrm{http}: / /$ bioinfo.bjmu.edu.cn/mirsnp/search/ \\
Spliceport & Spicing prediction & $\mathrm{http}: / /$ spliceport.cbcb.umd.edu/ \\
RNAfold & mRNA2 $2^{\text {ry }}$ structure & $\mathrm{http}: / /$ rna.tbi.univie.ac.at/cgi-bin/RNAfold.cgi \\
PLINK & SNP-SNP interaction & $\mathrm{http}: / /$ pngu.mgh.harvard.edu/ purcell/plink/ \\
SNAP & SNPs LD blocks & $\mathrm{https://www.broadinstitute.org/mpg/snap/ldsearch.php}$
\end{tabular}

Table 3. Shows association of different haplotypes of IL-4, IL-5 and IL-13 with malaria, (F-A=Frequency in affected, F-U= Frequency in unaffected)

\begin{tabular}{lccc}
\hline Haplotype & F-A & F-U & $p$-value \\
\hline GCG & 0.00 & 0.24 & 0.02 \\
GTG & 0.11 & 0.04 & 0.25 \\
ATG & 0.07 & 0.02 & 0.27 \\
GCT & 0.05 & 0.11 & 0.50 \\
ACT & 0.06 & 0.03 & 0.50 \\
GTT & 0.13 & 0.05 & 0.20 \\
ATT & 0.55 & 0.49 & 0.60 \\
\hline
\end{tabular}


Table 4. Transcription factors predicted by the different softwares

\begin{tabular}{|c|c|c|c|c|c|c|c|c|c|}
\hline & SNP function & & & RegulomeDF & & Ensemble & & & Regulatory \\
\hline Tool SNP & prediction & IsrSNP & $(\text { score })^{*}$ & rSNPBASE & Regulatory region & Alibaba2.1 & TFSEARCH & Potential score & Conservation \\
\hline rs734244 (IL-4) & - & - & POLR2A, GATA2, GATA1 4 & + & ENSR00001290157 & SP-1 & SP-1 & 0.19 & 0.004 \\
\hline rs1800474 (IL-5) & - & LM7 & - & - & - & - & - & 0.22 & 0.934 \\
\hline rs 1881457 (IL-13) & + & - & GATA1 (3a) & - & ENSR00001290150 & SP-1 & SP-1 & 0.00 & 0.000 \\
\hline rs2070874 (IL-4) & + & LM101 & $\operatorname{MAX}(2 b)$ & + & - & Ftz & Ftz & 0.09 & 0.725 \\
\hline
\end{tabular}

*RegulomeDB score:

Score: Supporting evidence

2b: TF binding + any motif + Dnase Footprint + Dnase peak

3a: $\quad$ TF binding + any motif + Dnase peak

4: $\quad$ TF binding + Dnase peak

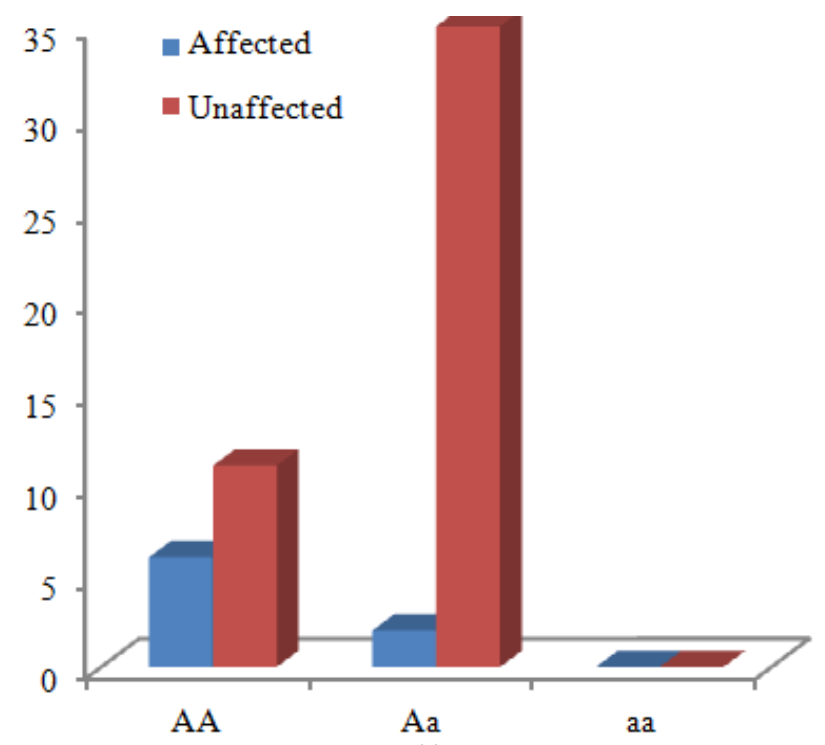

(a)

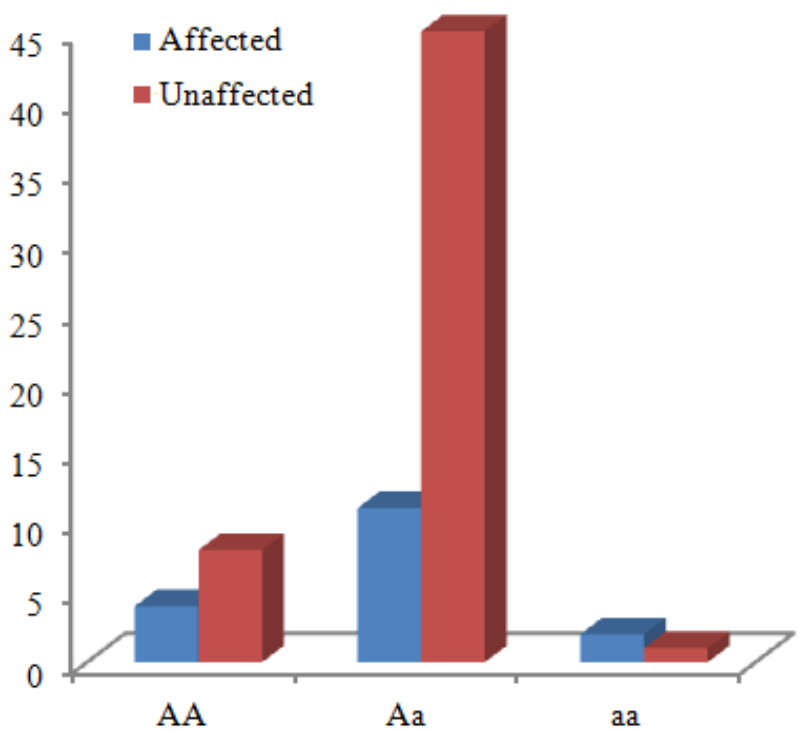

(b)

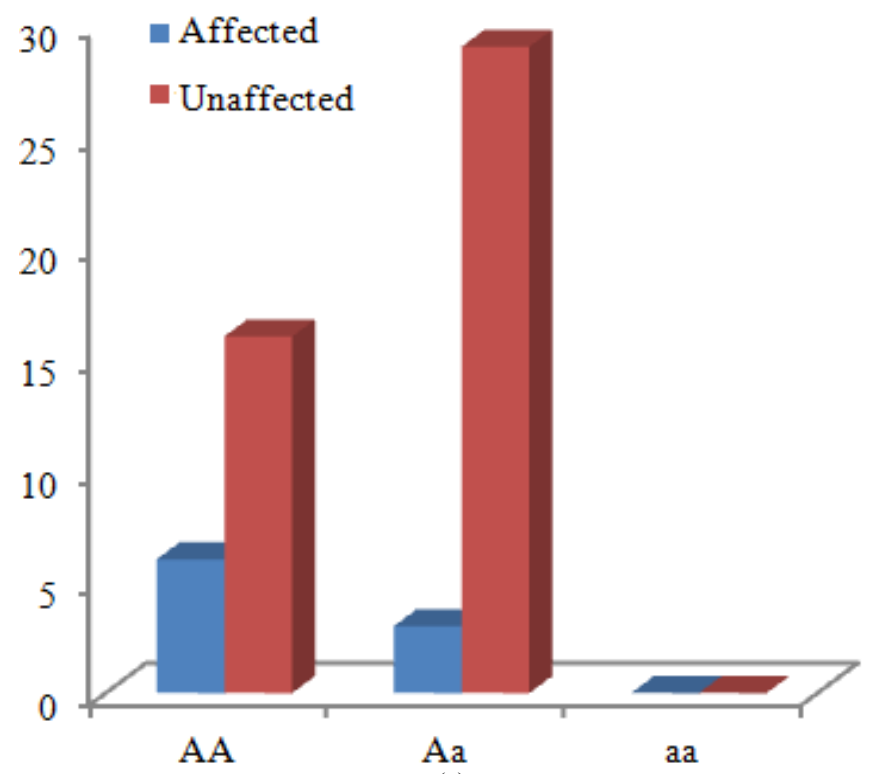

(c)

Fig. 1. (a) Frequency of malaria for different IL-5 rs 1800474 genotypes, odd ratio: 0.11, p-value $=0.008$, (b) Frequency of malaria for different IL-4 rs734244 genotypes, p-value $=0.11$, (c) Frequency of malaria for different IL-13 rs1881457 genotypes, odd ratio: 0.33 , $\mathrm{p}$-value $=0.24$ 


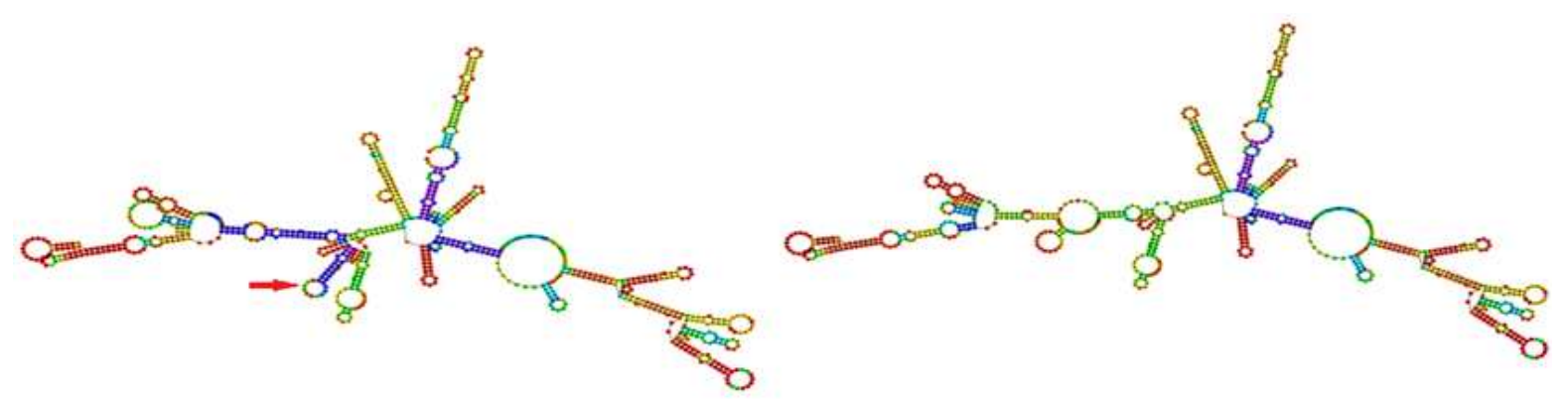

Fig. 2. To the left: The secondary structure of IL-5 mRNA, to the right: The structure of the same molecule with the variation rs1800474. Arrow indicates the site of the stem loop

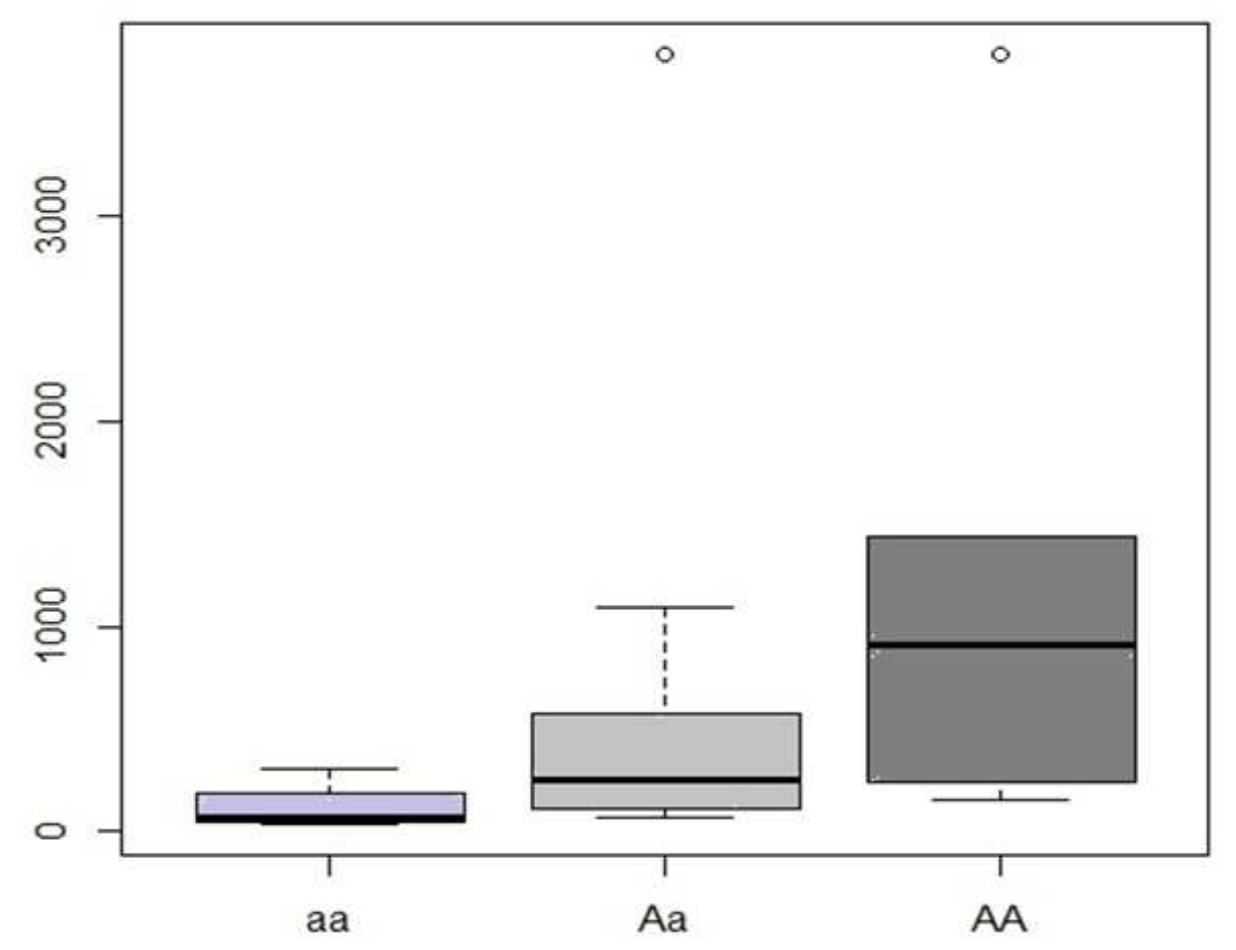

Fig. 3. The correlation between different IL-4 rs734244 genotypes and mean serum IgE level (IU/mL), (p-value=0.32)

In addition, rs1800474 was found to change the secondary structure of IL- 5 mRNA by changing a stem loop structure in the center of the molecule, Fig. 2. There was stepwise increase in mean serum IgE level from homozygous minor (aa) to heterozygous (Aa) and homozygous major (AA), p-value $=0.32$ Fig. 3. The latter genotype is associated with (A) allele which binds many transcription factors. In another unpublished data of ours we found that IL-4 mRNA level is twice that of the control (the samples were taken randomly from Hausa population).

Mir SNP predicted no result for miRNA binding sites and Splice Port predicted no effect for splicing variations. PLINK tool showed that there is no SNP to SNP interaction; every SNP has a unique effect.

\section{Discussion}

Visceral Leishmaniasis (VL) and malaria are two major parasitic diseases which overlap geographically and may co-exist in the same patients. Van den Bogaart et al. (2014) compared the analysis of cytokine profiles from co-and mono-infected patients and highlighted significant differences in the immune response mounted upon co-infection, confirming the ability of L. Donovani and $P$. falciparum to mutually interact at the immunological level. The study clearly revealed the positive role of TH1 cytokine response and TNF-alpha in both malaria and visceral leishmaniasis, but the role of TH2 cytokine response in co-infected individuals is yet to be determined (Van den Bogaart et al., 2014). Many studies revealed the positive role of $\mathrm{TH} 2$ cytokine 
response in the clearance of malaria but not visceral leishmaniasis (see below). The different cytokine TH2 response in these two diseases may result in variable pressures in $5 \mathrm{q} 31$ region in areas where malaria and visceral leishmaniasis are both endemic. In Koka village there was no evidence of overt co-infection of malaria and $\mathrm{VL}$, but occult infections remain to be shown.

In this study we suggested that the observed excess in heterozygosity in 5q31.1 region is due to unbalanced polymorphism (overdominance) between two major endemic diseases; malaria and visceral leishmaniasis. But the model cannot be simple as in the case of sickle cell anemia and malaria; because cytokine responses for different environmental triggers are very complex and many contradicting factors favor up or down regulations of different immunological responses, malaria favors a strong TH2 response; high level of IL-4 and IL-5 in malaria positive patients correlates with mild symptoms and rapid parasite clearance (Prakash et al., 2006; Troye-Blomberg et al., 1990). We failed to detect significant association between risk of malaria and serum IgE level ( $p$-value $=0.07$ ) probably due to the small sample size, but Interestingly, we found that the haplotype GCG of IL-4, IL-5 and IL-13 respectively which is associated with greater risk of malaria ( $p$-value $=0.02)$, is the haplotype associated with little/no transcription factor binding and decreased mRNA stability (i.e., low cytokine $\mathrm{TH} 2$ profile) as predicted by the different tools. On the other hand in visceral leishmaniasis $\mathrm{TH} 2$ response has negative outcome; it correlates with progressive lesions and disseminated leishmaniasis (Ribeiro-de-Jesus et al., 1998). In Sudan, showed that IL-4 contributes to the susceptibility of visceral leishmaniasis (Mohamed et al., 2013).

These two factors (malaria and VL) acting differently probably result in a $\mathrm{TH} 2$ cytokine response that is optimum for these strong forces and manifested in the form of excess heterozygosity for the SNPs that have direct effect on the expression, regulation and function of interleukins. There are probably other factors besides malaria and visceral leishmaniasis; like schistosomiasis, asthma and childhood respiratory infections acting on 5 q31 chromosomal region forming a complex interplay driving the locus towards excess heterozygosity. This illustrates how complex gene-environment interaction is and understanding it allows us to comprehend the factors that shaped human evolution both at molecular and phenotypic level.

\section{Conclusion}

Immunological interaction between malaria and visceral leishmaniasis is very complex, both diseases require strong cytokine $\mathrm{TH} 1$ response but for cytokine $\mathrm{TH} 2$ the story is different. SNPs in 5q31 which have direct role in the expression of interleukins are subject to balancing selection (heterozygosity) that drives the locus towards optimum response.

\section{Acknowledgement}

The authors wish to acknowledge the cooperation of Koka villagers and the EviMalar European Network of Excellence. The authors would like to thank Malaria GEN for providing a framework for the study, genotyping the samples and undertaking the quality control.

\section{Funding Information}

The authors acknowledge the Institute of endemic diseases, University of Khartoum, for providing the necessary funds and support for research purposes.

\section{Author's Contributions}

Mutaz Amin: Analysed the data and wrote and submitted the manuscript.

Abdelbadea Elhassan: Provided the raw data,

Kirk Rocket: Edited the manuscript

Muntasir Ibrahim: Revised and supervised the work.

\section{Ethics}

The study has been approved by the Ethics Review Committee of the Institute of Endemic Diseases, University of Khartoum. No ethical issues were aroused during or after publication of the manuscript.

\section{References}

Elhassan, A.A., A.A. Hussein, H.S. Mohamed, K. Rockett and D. Kwiatkowski et al., 2013. The 5q31 region in two African populations as a facet of natural selection by infectious diseases. Genetics, 49: 279-288. PMID: 23668094

Mohamed, H.S., M.E. Ibrahim, E.N. Miller, C.S. Peacock and E.A.G. Khalil et al., 2003. Genetic susceptibility to visceral leishmaniasis in The Sudan: linkage and association with IL4 and IFNGR1. Genes Immunity, 4: 351-355.

DOI: $10.1038 /$ sj.gene.6363977

Prakash, D., C. Fesel, R. Jain, P.A. Cazenave, G.C. Mishra and S. Pied, 2006. Clusters of cytokines determine malaria severity in Plasmodium falciparum-infected patients from endemic areas of Central India. J. Infect. Dis., 194: 198-207.

DOI: $10.1086 / 504720$

Ribeiro-de-Jesus, A., R.P. Almeida, H. Lessa, O. Bacellar and E.M. Carvalho, 1998. Cytokine profile and pathology in human leishmaniasis. Brazilian J. Med. Biol. Res., 31: 143-148.

DOI: $10.1590 / \mathrm{S} 0100-879 X 1998000100020$ 
Troye-Blomberg, M., E.M. Riley, L. Kabilan, M. Holmberg and H. Perlmann et al., 1990. Production by activated human $T$ cells of interleukin 4 but not interferon-gamma is associated with elevated levels of serum antibodies to activating malaria antigens. Proc. National Acad. Sci., 87: 5484-5488.

DOI: $10.1073 /$ pnas.87.14.5484
Van den Bogaart, E., A.B.A. Talha, M. Straetemans, P.F. Mens and E.R. Adams et al., 2014. Cytokine profiles amongst Sudanese patients with visceral leishmaniasis and malaria co-infections. BMC Immunol., 15: 1-10. DOI: 10.1186/1471-2172-15-16 